\title{
Applicability of the Nutrition Risk Screening 2002 Combined with a Patient-Generated Subjective Global Assessment in Patients with Nasopharyngeal Carcinoma
}

This article was published in the following Dove Press journal:

Cancer Management and Research

\author{
Xiaoxian Pan ${ }^{1,}$ \\ Caihong Wangl, \\ Rong $\mathrm{Li}^{2}$ \\ Li Su ${ }^{1,3,4}$ \\ Mingwei Zhang 1,3,4 \\ Chuanshu Cai ${ }^{1,3,4}$ \\ Shiping Liu' \\ Weijian Zhang ${ }^{1,3,4}$ \\ Jihong Song ${ }^{2}$ \\ Jinsheng Hong (1, 3,4 \\ 'Department of Radiotherapy, First \\ Affiliated Hospital of Fujian Medical \\ University, Fuzhou, Fujian, People's \\ Republic of China; ${ }^{2}$ The School of \\ Nursing, Fujian Medical University, \\ Fuzhou, Fujian, People's Republic of \\ China; ${ }^{3}$ Key Laboratory of Radiation \\ Biology (Fujian Medical University), Fujian \\ Province University, Fuzhou, Fujian, \\ People's Republic of China; ${ }^{4}$ Fujian \\ Provincial Key Laboratory of Precision \\ Medicine for Cancer, Fuzhou, Fujian, \\ People's Republic of China
}

*These authors contributed equally to this work

\begin{abstract}
Purpose: This study aims to explore the applicability of the Nutrition Risk Screening 2002 (NRS2002) tool in screening nutritional risk and the Patient-Generated Subjective Global Assessment (PG-SGA) in determining nutrition status in nasopharyngeal carcinoma (NPC) patients.

Materials and Methods: NRS2002 and PG-SGA were simultaneously applied to evaluate the nutritional status of NPC patients before induction chemotherapy, as well as before and after radiotherapy. The PG-SGA results were considered golden standard in evaluating nutrition status, and the ROC curve value and Youden index were applied to analyze NRS2002 effectiveness in screening nutritional risk.

Results: A total of 102 NPC patients were included in this study. Patients with an NRS2002 score $<3$ and PG-SGA score $\geq 4$ accounted for 5.3\% (5/95), 19.6\% (18/92) and 94.8\% (36/38) at the time before induction chemotherapy, before radiotherapy and at the end of radiotherapy, respectively. The cut-off values of NRS2002 scores all $<2$ corresponded to the maximum Youden index at the three procedural times. And the area under curve (AUC) were $0.598(P=0.390), 0.665(P=0.015)$ and $0.940(P=0.034)$, respectively. At the end of radiotherapy, NRS2002 scores of $<3$ and $<2$ were used as cut-off values for nutritional risk screening, respectively. Additionally, the malnutrition-missed detection rates were $36.0 \%$ and $12.0 \%\left(\chi^{2}=15.789 ; P<0.001\right)$.

Discussion: NRS2002 nutritional risk screening combined with the PG-SGA nutritional assessment has certain applicability in NPC. NRS2002 score $\geq 2$ can be considered as a new cut-off point for nutritional assessment.
\end{abstract}

Keywords: nasopharyngeal carcinoma, nutritional risk screening, nutrition assessment, NRS2002, PG-SGA

\section{Introduction}

Nasopharyngeal carcinoma (NPC) is a common tumor in southern China. A total of 129,079 new cases of NPC were diagnosed worldwide in 2018. ${ }^{1}$ Head and neck tumors, including NPC, are often accompanied by malnutrition. ${ }^{2}$

Therapeutic toxicity that significantly affects intake during radiotherapy and chemotherapy can further increase malnutrition in patients. ${ }^{3,4}$ In addition, malnutrition in NPC patients is usually associated with a poorer prognosis. ${ }^{5}$ Studies have confirmed that early nutritional management can reduce weight loss ${ }^{6}$ and improve both the quality
Correspondence: Jinsheng Hong

Email13799375732@I63.com
Cancer Management and Research 2020:I 822 I-8227

8221

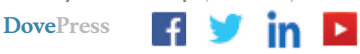


of life and prognosis in patients. ${ }^{7-9}$ Therefore, timely nutritional intervention on detecting malnutrition is an essential component of anti-tumor therapy of NPC patients.

According to the American Society for Parenteral and Enteral Nutrition (ASPEN) clinical guidelines (version 2011), hospitalized patients should first undergo a nutritional risk screening. Then, patients with nutritional risks should undergo a nutritional assessment to provide a basis for formulating nutritional intervention plans. ${ }^{10}$

Currently, the commonly used nutritional screening includes the Nutrition Risk Screening 2002 (NRS2002), and the Malnutrition Universal Screening Tool (MUST). ${ }^{11}$ NRS2002 is most commonly used for hospitalized patients as recommended by the European Society for Parenteral and Enteral Nutrition (ESPEN), ${ }^{12}$ which can also be used in patients with malignant tumors. ESPEN recommended that it should be performed within 24 hours of the patients' admission. ${ }^{13}$

Nutritional evaluation resources include the PatientGenerated Subjective Global Assessment (PG-SGA) tool, the Mini Nutritional Assessment (MNA) tool. ${ }^{14}$ The American Dietetic Association (ADA) has recommended the use of PG-SGA ${ }^{15}$ as the preferred nutritional assessment tool for cancer patients. ${ }^{10}$ PG-SGA can more accurately identify malnourished patients than NRS2002 and is often used as the golden standard for nutritional assessment of cancer patients, ${ }^{16,17}$ although both tools are applied in the clinic. However, there are only relatively few clinical studies that have explored the applicability of NRS2002 when combined with the PG-SGA scale in patients with NPC.

\section{Materials and Methods}

\section{Patients}

This is a retrospective cohort study to evaluate the nutritional status of NPC patients that received radiotherapy in the Department of Radiotherapy of the First Affiliated Hospital of Fujian Medical University from February 2018 to July 2019.

All enrolled patients were treated with radiotherapy and chemotherapy in accordance with the NPC diagnosis and treatment standards. ${ }^{18}$ This study was approved by the relevant local institutional review boards for human research at Fujian Medical University, and all participants provided written informed consent.

Inclusion criteria included: (i) pathological diagnosis of NPC; (ii) being conscious, with a certain language communication competence; (iii) those that received radical radiotherapy and induction chemotherapy; and (iv) a willingness to cooperate with nutritional risk screening and the nutritional status assessment. And exclusion criteria included: (i) patients that presented with other malignant tumors in the past; (ii) those patients that did not complete the three nutritional evaluations, including immediately prior to induction chemotherapy, and immediately prior to, and following radiotherapy; and (iii) those that did not cooperate or could not cooperate with nutrition evaluation investigators.

\section{Nutritional Evaluation Method}

NRS2002 was used to screen the nutritional risk of NPC patients. A score $\geq 3$ was considered to have a nutritional risk. PG-SGA was used to assess the nutrition status of patients. Score of 0 to 1 was set as one of good nutrition, score of 2 to 3 was classified as being evident of a suspicious malnutrition, score of 4 to 8 was classified as moderate malnutrition, and a score $\geq 9$ was classified as having severe malnutrition. Nutritional risk screening and nutritional assessments were performed by trained professionals using the NRS2002 and PG-SGA criteria for patients with NPC before induction chemotherapy, as well as prior to, and following radiotherapy.

\section{Statistical Methods}

Data were analyzed using SPSS statistical analysis software (version 23.0; International Business Machines Corporation, USA). Not all data were normally distributed, and baseline characteristics were expressed as the frequency or median. SAS 9.4 (Statistical Analysis System, USA) was used to analyze whether the nutritional risk or the malnutrition positive rate varied at different times through the generalized linear mixed-effects model, and whether the missed detection rate of NRS2002 varied at different time-points. The evaluated results of the PG-SGA scale were used as diagnostic criteria for malnutrition, and the ROC curve was used to analyze the effectiveness of NRS2002 in screening nutritional risks. The $Z$-test was used to compare differences in sensitivity at different cut-off points. The Chi-square test or the exact probability method was used to compare the differences of missed detection rates among different groups. An alpha value of $P<0.05$ infers a statistically significant difference.

\section{Results}

\section{Patient Information}

Herein, 105 NPC patients met the enrollment criteria. Three patients were excluded since they had not been evaluated on 
all available scales. Some 102 eligible patients with NPC were included, with a median age of 48 years. There were 73 males $(71.57 \%)$ and 29 females (28.43\%). Clinical staging (ie, in accordance with the AJCC 8th edition of nasopharyngeal carcinoma staging) was as follows: stage II: 4 cases; stage III: 47 cases; and stage IV: 51 cases.

\section{Comparison of NRS2002 and PG-SGA Scores}

Results of the NRS2002 and PG-SGA scores before chemotherapy, as well as prior to, and at the end of radiotherapy are shown in Table 1. It is worth noting that as treatment progressed, the proportion of patients with nutritional risk and moderate or severe malnutrition increased significantly. The results of generalized linear mixed model analysis showed that both positive diagnosis rates of NRS2002 $(P<0.001)$ and PG-SGA $(P<0.001)$ were significantly different when determined at the three measured time-points. The number of missed malnutrition patients (ie, a NRS2002 score $<3$ and PG-SGA score $\geq 4$ ) also increased significantly (Table 2). The generalized linear mixed model analysis showed that the missed malnutrition detection rate for NPC patients at different time-points was significantly different $(P<0.001)$, and was the highest at the time after induction chemotherapy and before radiotherapy.

\section{Results of NRS2002 Screening Nutritional Risk Effectiveness Analysis}

Taking the PG-SGA nutritional assessment result as the golden standard, analysis of effectiveness of NRS2002 in screening nutritional risks showed that the area under the ROC curve of the NRS2002 score when measured for a total of 306 times across 102 patients at three timepoints was $0.842(P<0.05)$. When a cut-off point of 1.5 (NRS 2002 score $<2$ ) was used for the NRS2002 score, the

Table I Comparison of NRS2002 and PG-SGA Scores in 102 Patients with NPC

\begin{tabular}{|l|l|l|l|l|}
\hline \multirow{2}{*}{ Time } & \multicolumn{2}{|l|}{ NRS2002 } & \multicolumn{2}{l|}{ PG-SGA } \\
\cline { 2 - 5 } & $<3$ & $\geq 3$ & $<4$ & $\geq \mathbf{4}$ \\
\hline Before CT & $95(93.1 \%)$ & $7(6.9 \%)$ & $95(93.1 \%)$ & $7(6.9 \%)$ \\
After CT & $92(90.2 \%)$ & $10(9.8 \%)$ & $78(76.5 \%)$ & $24(23.5 \%)$ \\
At the End of RT & $38(37.3 \%)$ & $64(62.8 \%)$ & $2(2.0 \%)$ & $100(98.0 \%)$ \\
Total & $225(73.5 \%)$ & $81(26.5 \%)$ & $175(57.2 \%)$ & $131(42.8 \%)$ \\
\hline
\end{tabular}

Abbreviations: Before $\mathrm{CT}$, before induction chemotherapy; After $\mathrm{CT}$, after induction chemotherapy before radiotherapy; RT, radiotherapy.
Table 2 Number of Missed Malnutrition Patients in 102 Patients with NPC (\%)

\begin{tabular}{|l|l|l|l|l|}
\hline \multirow{2}{*}{ Time } & \multicolumn{2}{|l|}{$\begin{array}{l}\text { NRS2002 } \\
\text { Score<3 }\end{array}$} & $\begin{array}{l}\text { PG-SGA } \\
\text { Score } \geq 4 \text { (in } \\
\text { All Patients) }\end{array}$ & $\begin{array}{l}\text { Missed } \\
\text { Malnutrition } \\
\text { Rate }\end{array}$ \\
\cline { 2 - 4 } & $\begin{array}{l}\text { PG- } \\
\text { SGA } \\
\text { Score } \\
<4\end{array}$ & $\begin{array}{l}\text { PG- } \\
\text { SGA } \\
\text { Score } \geq\end{array}$ & & \\
& $\mathbf{4}$ & & \\
\hline $\begin{array}{l}\text { Before CT } \\
\text { After CT }\end{array}$ & 90 & 5 & 7 & $71.4 \%(5 / 7)$ \\
At the End of RT & 24 & 18 & 24 & $75.0 \%(18 / 24)$ \\
Total & 2 & 36 & 100 & $36.0 \%(36 / 100)$ \\
\hline
\end{tabular}

Abbreviations: Before $\mathrm{CT}$, before induction chemotherapy; After $\mathrm{CT}$, after induction chemotherapy before radiotherapy; RT, radiotherapy.

maximum Youden index was 0.672 , and the sensitivity and specificity were $76.3 \%$ and $90.9 \%$, respectively.

In addition, while 1.5 was used as the cut-off point of the NRS2002 score, all of the Youden indices attained a maximum number at three different time-points (see Figure 1 and Table 3). The area under the ROC curve before induction chemotherapy, prior to, and at the end of radiotherapy were $0.598(P=0.390), 0.665(P=0.015)$, and 0.940 $(P=0.034)$, respectively. Furthermore, $Z$-test analyses, which were used to compare two cut-off points, showed no significant statistical differences in sensitivity when measured before the induction of chemotherapy and radiotherapy, respectively $(P>0.05$ for $Z=0.65$ and $Z=1.23$ ). The sensitivity showed statistically significant difference when measured at the end of radiotherapy $(Z=7.18, P<0.05)$.

\section{Comparison of the Missed Malnutrition Detection Rate on Applying an NRS2002 Score of $<2$ or $<3$ as the Cut-off Value}

The Chi-square test was used to compare the missed malnutrition diagnostic rates under the two bounded values for patients with an NRS2002 score between $<2$ and $<3$. This result showed that the missed rate for the 102 studied patients was $71.4 \%$ vs $71.4 \%(P=1.000), 58.3 \%$ vs $75.0 \%\left(\chi^{2}=1.50\right.$; $P=0.221)$ and $12.0 \%$ vs $36.0 \%\left(\chi^{2}=15.789 ; P=0.000\right)$ when determined before the induction of chemotherapy, as well as prior to, and at the end of radiotherapy, respectively.

\section{Discussion}

At present, the majority of cancer patients visited are in the middle and late stages. Given the universality of malnutrition in cancer populations, as well as the serious consequences of malnutrition, nutritional therapy should be the basis of conventional means and tumor therapy in 


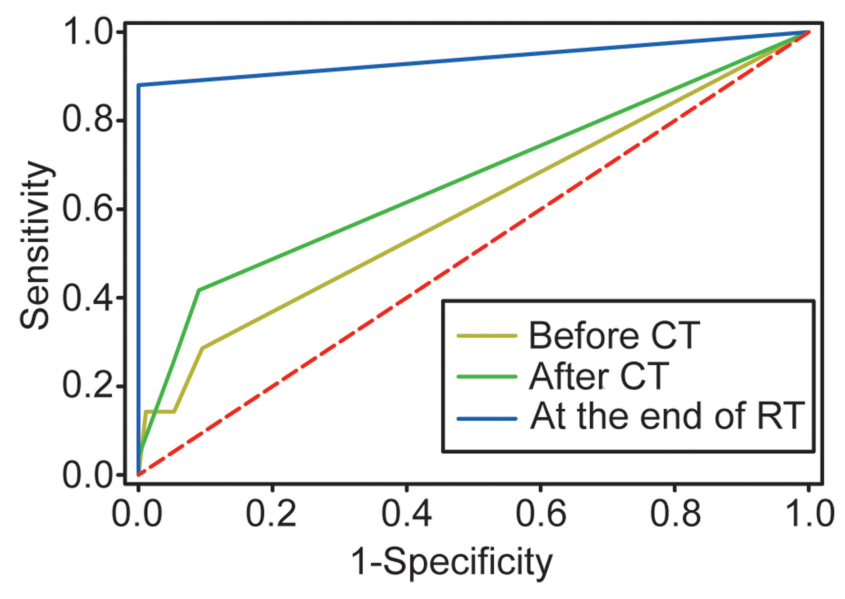

Figure I ROC curve analysis of 102 patients with NPC using the NRS2002 scale to screen nutritional risk. The area under the ROC curve before induction chemotherapy, after induction chemotherapy, and at the end of radiotherapy were $0.598(P=0.390)$, $0.665(P=0.015)$, and $0.940(P=0.034)$ respectively.

Abbreviations: Before $\mathrm{CT}$, before induction chemotherapy; After $\mathrm{CT}$, after induction chemotherapy before radiotherapy; RT, radiotherapy.

treating patients with tumors. For a reasonable nutrition therapy, first we need to assess the nutritional status of patients. Nutritional assessment aims to identify patients with malnutrition and determine the target of nutritional therapy, so as to ensure the reasonable application of nutritional therapy and prevent the insufficient and excessive application of nutritional therapy. In addition, during the nutrition treatment process, it needs to be re-evaluated continuously in order to adjust the treatment plan in time.

This study found that patients with NPC have a nutritional risk of only $26.5 \%$ when screened by NRS2002. After having been assessed by PG-SGA under conditions of moderate to severe malnutrition, the nutritional risk was as high as $42.8 \%$. Compared with an NRS2002 score $<3$ as the threshold for the presence or absence of nutritional risk, we found that when using an NRS2002 score $<2$ as the threshold, the screening sensitivity was improved, the rate of missed malnutrition detection was reduced, and patients could be prevented from missing an opportunity for timely nutritional intervention. Therefore, it is recommended that an NRS2002 score $<2$ should be used as the cut-off value to determine whether NPC patients enter the nutritional assessment path.

In this study, we found that the score items of NRS2002 screening in most patients were normal while those of PGSGA showing malnutrition were mainly distributed in the self-evaluation items of PG-SGA, including dietary intake, patient symptoms and physical examination. Kubrak ${ }^{19}$ also confirmed that dietary intake was related to weight loss and prognosis of patients with head and neck cancer.

NRS2002 is the first nutritional risk screening scale in the world that is based on empirical evidence-based medical evidence. Its nutritional risk and cut-off values are founded on comprehensive research and evaluation of all inpatients (including patients with liver cirrhosis, gastrointestinal surgery, cancer, and infections, etc.). However, there is no individual score for nasopharyngeal carcinoma in the NRS2002 scoring project, which leads to poor performance of NRS2002 in the nutritional risk screening for nasopharyngeal cancer patients.

Nasopharyngeal carcinoma has special characteristics. At the initial diagnosis, the main symptoms of NPC are sucking bloody sputum and a neck mass, where eating is generally normal. After induction chemotherapy, systemic side-effects including nausea, vomiting, fatigue and other discomfort that can collectively affect appetite and gastrointestinal absorption are noted, which are the main causes of malnutrition in NPC patients. ${ }^{20,21}$ In addition, combined chemo-radiotherapy can aggravate oral mucositis with damage to the taste buds, and the salivary gland tissues, which collectively makes it difficult for patients to eat, and with a noticeably decreased appetite conditions that further lead to reduced nutritional intake. This is concordant with

Table 3 Efficacy Analysis of Different Cut-off Values of NRS2002 Scale for Nutritional Risk Screening in NPC Patients

\begin{tabular}{|c|c|c|c|c|c|c|}
\hline Time & NRS Cut-Off value & Sensitivity & Specificity & Maximum Youden Index & AUC & $P$ value \\
\hline Before CT & $\begin{array}{l}1.5(<2) \\
2.5(<3)\end{array}$ & $\begin{array}{l}28.6 \% \\
14.3 \%\end{array}$ & $\begin{array}{l}90.5 \% \\
94.7 \%\end{array}$ & $\begin{array}{l}0.191 \\
0.090\end{array}$ & $59.8 \%$ & 0.390 \\
\hline After CT & $\begin{array}{l}1.5(<2) \\
2.5(<3)\end{array}$ & $\begin{array}{l}41.7 \% \\
25.0 \%\end{array}$ & $\begin{array}{l}91.0 \% \\
94.9 \%\end{array}$ & $\begin{array}{l}0.327 \\
0.199\end{array}$ & $66.5 \%$ & 0.015 \\
\hline At the End of RT & $\begin{array}{l}1.5(<2) \\
2.5(<3)\end{array}$ & $\begin{array}{l}88.0 \% \\
55.0 \%\end{array}$ & $\begin{array}{l}100 \% \\
100 \%\end{array}$ & $\begin{array}{l}0.880 \\
0.550\end{array}$ & $94.0 \%$ & 0.034 \\
\hline
\end{tabular}

Abbreviations: NRS, Nutrition Risk Screening 2002; AUC, area under the curve; Before CT, before induction chemotherapy; After CT, after induction chemotherapy before radiotherapy; RT, radiotherapy. 
our results that there were significant differences in the NRS2002 and PG-SGA aligned nutritional evaluation before and after induction chemotherapy, and at the end of radiotherapy, observations that were in addition to the significant increase in moderate and severe malnutrition rates after chemotherapy and radiotherapy.

In a large-scale study of 3232 patients with NPC by Hao Peng, ${ }^{22}$ it was found that the threshold value of the conventional NRS2002 scale may not be directly related to NPC, and correlations with the prognosis can be better obtained by revising the cut-off value for both age and observed weight loss of the patients. The results of this study showed that NRS2002 nutritional screening and PGSGA nutritional evaluation had a certain applicability in NPC patients. However, by adjusting the threshold value, we found that using a NRS2002 score of $<2$ as the cut-off point could improve the sensitivity in detecting the malnutrition rates.

It is worth mentioning that the positive diagnosis rate of nutritional assessment of patients with NPC at different times varies and shows statistical differences, indicating that the nutritional status of patients with NPC at different treatment stages is also different. In addition, the missed detection rates of NRS at different treatment stages also showed statistical differences. In this study, we found that the AUC of using the NRS2002 score $<2$ as the cutoff point at the end of radiotherapy was as high as $94.0 \%$. And the sensitivity with the NRS2002 score $<2$ as the cutoff point was significantly better than that with the NRS2002 score $<3$ as the cutoff point. Besides, the missed detection rate of these two point was statistically different, indicating that the model (NRS $<2$ as the cutoff point) had a good predictive ability at the end of radiotherapy. However, we found that although the sensitivity with NRS2002 score $<2$ as the cutoff point was greater than NRS $<3$ before induction chemotherapy and after induction chemotherapy before radiotherapy, no statistical difference between the two stages was observed. This may be due to the fact that most patients with NPC had a better nutritional status and fewer cases of malnutrition in these two stages of treatment.

In this study, the NRS2002 score $<3$ accounted for $14.3 \%$ and the specificity was $94.7 \%$ before induction chemotherapy (when admitted to our department), indicating that the screening sensitivity was low. This is similar to the work of $\mathrm{Du}^{23}$ that reported a comparison of two nutritional evaluation tools (ie, albumin levels that were lower than the normal value were judged as the standard of nutritional risk) in 927 inpatients with a tumor sensitivity (sensitivity $43.13 \%$; specificity $82.16 \%$ ) of the NRS2002 approach being significantly lower than that provided by PG-SGA (sensitivity 93.78\%; specificity 21.80\%). This results showed that NRS2002 is more likely to miss a detection than PG-SGA would do.

In previous researches, some scholars believed that the nutritional assessment should be routinely performed even if the nutrition risk screening was negative for patients who are with cancer, old or are critically ill. This is based on the research of Bauer, ${ }^{24}$ Yang, ${ }^{25}$ which confirmed that NRS2002 had a high false-negative rate in some common cancer nutritional screening results, while PGSGA was more sensitive in the detection of cancer than NRS2002, such as in the settings of lung, esophageal, gastric, liver cancer, and so on. This is consistent with our result that the positive rate of NRS2002 was significantly lower than that of PG-SGA. However, in these studies, there were few reported cases of NPC, and only 6 cases of NPC were reported by Yang. ${ }^{25}$ In addition, Orell-Kotikangas, in the study of head and neck cancer, showed that NRS2002 score $\geq 2$ had high specificity and sensitivity of $78.8 \%$ and $96.9 \%$ for PG-SGA $\geq 4$, respectively. However, the study was based on all head and neck tumors, not nasopharyngeal cancer, and only 7 cases of NPC were reported. ${ }^{26}$

Nevertheless, the evaluated content of the PG-SGA scale is subjective and takes a long time to complete. Balstad ${ }^{27}$ proposed that, since part of the scale based its scoring on subjective feelings of the patients, some wording could lead to an incorrect evaluation of the patients, which needs more suitable standardized rules of evaluation. In recent years, many studies have found that malnutrition in NPC patients is also associated with multiple indicators including biomarkers like albumin and hemoglobin. ${ }^{28}$ Jin et al proposed that pre-albumin levels are predictive markers of weight loss in NPC patients. ${ }^{29}$ There are also scholars who have proposed other effective indicators for a nutritional evaluation, ${ }^{30}$ including the comprehensive nutritional index (CNI) proposed by Deng et al, ${ }^{31}$ and bioelectrical impedance analysis (BIA) to objectively measure body composition and nutritional status of NPC patients suggested by Ding et al, ${ }^{32}$ as well as grip strength measurements recommended by $\mathrm{McNicholl}{ }^{33}$

Interestingly, Gabrielson et $\mathrm{al}^{34}$ also proposed that a simplified version of PG-SGA (ie, the abridged scored Patient-Generated Subjective Global Assessment or abPGSGA) could be used as a nutritional screening tool for cancer out-patients. Thus, it can be seen that further 
enrichment of the nutritional evaluation scale for NPC patients, or indeed the search for a more objective and convenient nutritional evaluation index in the setting of NPC still requires further exploration.

The shortcomings of this study are that the sample size was relatively too small and it was a single-center experience. It is expected that further multi-central studies with expanded sample or cohort size will be conducted to verify the study results and outcomes reported herein.

\section{Conclusions}

This study found that NRS2002 nutrition risk screening combined with the PG-SGA nutrition assessment has certain applicability for patients with nasopharyngeal carcinoma. Using a NRS2002 score $\geq 3$ to assess nutritional risk exhibits a low level of sensitivity. It is recommended that investigators should use NRS2002 score $\geq 2$ as a new cut-off point for distinguishing the malnutrition individuals.

\section{Data Sharing Statement}

We declare that the materials described in this manuscript, including all relevant raw data, will be freely available to any scientist wishing to use them for non-commercial purposes, without breaching participant confidentiality.

\section{Ethics Approval and Informed Consent}

This study was approved by the Ethics Committee of First Affiliated Hospital of Fujian Medical University, which was in accordance with the Declaration of Helsinki. The approval number is [2015]084-1, and written informed consent was obtained from each study subject prior to enrollment.

\section{Acknowledgments}

We were grateful to Professor Tian for her guidance on statistical methods, and we also thank the editors and reviewers for their comments.

\section{Author Contributions}

All authors made a significant contribution to the work reported, whether that is in the conception, study design, execution, acquisition of data, analysis and interpretation, or in all these areas; took part in drafting, revising or critically reviewing the article; gave final approval of the version to be published; have agreed on the journal to which the article has been submitted; and agree to be accountable for all aspects of the work. First author: Xiaoxian Pan and Caihong Wang should be considered joint first author.

\section{Funding}

This project supported by the Natural Science Foundation of Fujian Province, China [Grant No. 2017J01815].

\section{Disclosure}

The authors declare that they have no real or perceived conflicts of interest for this work.

\section{References}

1. Bray F, Ferlay J, Soerjomataram I, Siegel RL, Torre LA, Jemal A. Global cancer statistics 2018: GLOBOCAN estimates of incidence and mortality worldwide for 36 cancers in 185 countries. CA Cancer J Clin. 2018;68(6):394-424. doi:10.3322/caac.21492

2. Arends J, Baracos V, Bertz H, et al. ESPEN expert group recommendations for action against cancer-related malnutrition. Clin Nutr. 2017;36(5):1187-1196. doi:10.1016/j.clnu.2017.06.017

3. Lees J. Incidence of weight loss in head and neck cancer patients on commencing radiotherapy treatment at a regional oncology centre. Eur J Cancer Care (Engl). 1999;8(3):133-136. doi:10.1046/j.13652354.1999.00156.x

4. Ng K, Leung SF, Johnson PJ, Woo J. Nutritional consequences of radiotherapy in nasopharynx cancer patients. Nutr Cancer. 2004;49 (2):156-161. doi:10.1207/s15327914nc4902_6

5. Li G, Gao J, Liu ZG, et al. Influence of pretreatment ideal body weight percentile and albumin on prognosis of nasopharyngeal carcinoma: long-term outcomes of 512 patients from a single institution. Head Neck. 2014;36(5):660-666. doi:10.1002/hed.23357

6. Capuano G, Grosso A, Gentile PC, et al. Influence of weight loss on outcomes in patients with head and neck cancer undergoing concomitant chemoradiotherapy. Head Neck. 2008;30(4):503-508. doi: $10.1002 /$ hed.20737

7. Wei J, Wu J, Meng L, et al. Effects of early nutritional intervention on oral mucositis in patients with radiotherapy for head and neck cancer. QJM. 2020;113(1):37-42. doi:10.1093/qjmed/hcz222

8. Huang JF, Sun RJ, Jiang WJ, et al. Systematic nutrition management for locally advanced nasopharyngeal carcinoma patients undergoing radiotherapy. Onco Targets Ther. 2019;12:8379-8386. doi:10.2147/ OTT.S213789

9. Meng L, Wei J, Ji R, et al. Effect of early nutrition intervention on advanced nasopharyngeal carcinoma patients receiving chemoradiotherapy. J Cancer. 2019;10(16):3650-3656. doi:10.7150/ jca. 33475

10. Mueller C, Compher C, Ellen DM; American Society for P, Enteral Nutrition Board of D. A.S.P.E.N. clinical guidelines: nutrition screening, assessment, and intervention in adults. JPEN J Parenter Enteral Nutr. 2011;35(1):16-24. doi:10.1177/0148607110389335

11. Eldh AC, Ekman I, Ehnfors M. Considering patient non-participation in health care. Health Expect. 2008;11(3):263-271. doi:10.1111/ j.1369-7625.2008.00488.x

12. Kondrup J, Rasmussen HH, Hamberg O, Stanga Z. Nutritional risk screening (NRS 2002): a new method based on an analysis of controlled clinical trials. Clin Nutr. 2003;22(3):321-336. doi:10.1016/ S0261-5614(02)00214-5

13. Kondrup J, Allison SP, Elia M, Vellas B, Plauth M. ESPEN guidelines for nutrition screening 2002. Clin Nutr. 2003;22(4):415-421. doi:10.1016/S0261-5614(03)00098-0 
14. Guigoz Y, Lauque S, Vellas BJ. Identifying the elderly at risk for malnutrition. The mini nutritional assessment. Clin Geriatr Med. 2002;18(4):737-757. doi:10.1016/S0749-0690(02)00059-9

15. Ottery FD. Definition of standardized nutritional assessment and interventional pathways in oncology. Nutrition. 1996;12(1):S15-19. doi:10.1016/0899-9007(95)00067-4

16. Van BP, Thuy LN, Thanh HNT, et al. Comparison of novel, bach mai boston tool (BBT) and the patient-generated subjective global assessment (PG-SGA) for oncology inpatients. Cancer Control. 2019;26 (1):1073274819863767. doi:10.1177/1073274819863767

17. Abbott J, Teleni L, McKavanagh D, Watson J, McCarthy A, Isenring E. A novel, automated nutrition screening system as a predictor of nutritional risk in an oncology day treatment unit (ODTU). Support Care Cancer. 2014;22(8):2107-2112. doi:10.1007/s00520-014-2210-7

18. Hong JS, Hua YJ, Su L, et al. Modified-nutrition index is a significant prognostic factor for the overall survival of the nasopharyngeal carcinoma patients who undergo intensity-modulated radiotherapy. Nutr Cancer. 2017;69(7):1011-1018. doi:10.1080/01635581.2017.1359311

19. Kubrak C, Martin L, Gramlich L, et al. Prevalence and prognostic significance of malnutrition in patients with cancers of the head and neck. Clin Nutr. 2019;39(3):901-909.

20. Li G, Jiang XY, Qiu B, Shen LJ, Chen C, Xia YF. Vicious circle of acute radiation toxicities and weight loss predicts poor prognosis for nasopharyngeal carcinoma patients receiving intensity modulated radiotherapy. J Cancer. 2017;8(5):832-838. doi:10.7150/jca.17458

21. Hong JS, Tian J, Han QF, Ni QY. Quality of life of nasopharyngeal cancer survivors in China. Curr Oncol. 2015;22(3):e142-147. doi: $10.3747 / \operatorname{co} .22 .2323$

22. Peng H, Chen BB, Tang LL, et al. Prognostic value of nutritional risk screening 2002 scale in nasopharyngeal carcinoma: a large-scale cohort study. Cancer Sci. 2018;109(6):1909-1919. doi:10.1111/cas.13603

23. Du H, Liu B, Xie Y, et al. Comparison of different methods for nutrition assessment in patients with tumors. Oncol Lett. 2017;14 (1):165-170. doi:10.3892/ol.2017.6154

24. Bauer J, Capra S, Ferguson M. Use of the scored patient-generated subjective global assessment (PG-SGA) as a nutrition assessment tool in patients with cancer. Eur J Clin Nutr. 2002;56(8):779-785. doi:10.1038/sj.ejen.1601412
25. Yang JJYK, Huang YB, Yu M, et al. Comparison of NRS 2002 and PG-SGA for the assessment of nutritional status in cancer patients. Biomed Res. 2016;27(4):1178-1182.

26. Orell-Kotikangas H, Österlund P, Saarilahti K, Ravasco P, Schwab U, Mäkitie AA. NRS-2002 for pre-treatment nutritional risk screening and nutritional status assessment in head and neck cancer patients. Support Care Cancer. 2015;23(6):1495-1502. doi:10.1007/s00520014-2500-0

27. Balstad TR, Bye A, Jenssen CR, Solheim TS, Thoresen L, Sand K. Patient interpretation of the patient-generated subjective global assessment (PG-SGA) short form. Patient Prefer Adherence. 2019;13:1391-1400. doi:10.2147/PPA.S204188

28. Li XY, Sun XS, Liu SL, et al. The development of a nomogram to predict post-radiation necrosis in nasopharyngeal carcinoma patients: a large-scale cohort study. Cancer Manag Res. 2019;11:6253-6263. doi:10.2147/CMAR.S197841

29. Jin T, Li KX, Li PJ, et al. An evaluation of nutrition intervention during radiation therapy in patients with locoregionally advanced nasopharyngeal carcinoma. Oncotarget. 2017;8(48):83723-83733. doi:10.18632/oncotarget.19381

30. Saroul N, Pastourel R, Mulliez A, et al. Which assessment method of malnutrition in head and neck cancer? Otolaryngol Head Neck Surg. 2018;158(6):1065-1071. doi:10.1177/0194599818755995

31. Deng J, He Y, Sun XS, et al. Construction of a comprehensive nutritional index and its correlation with quality of life and survival in patients with nasopharyngeal carcinoma undergoing IMRT: a prospective study. Oral Oncol. 2019;98:62-68. doi:10.1016/j.oraloncology.2019.09.014

32. Ding H, Dou S, Ling Y, et al. Longitudinal body composition changes and the importance of fat-free mass index in locally advanced nasopharyngeal carcinoma patients undergoing concurrent chemoradiotherapy. Integr Cancer Ther. 2018;17(4):1125-1131. doi:10.1177/1534735418807969

33. McNicholl T, Dubin JA, Curtis L, et al. Handgrip strength, but not 5-meter walk, adds value to a clinical nutrition assessment. Nutr Clin Pract. 2019;34(3):428-435. doi:10.1002/ncp.10198

34. Gabrielson DK, Scaffidi D, Leung E, et al. Use of an abridged scored patient-generated subjective global assessment (abPG-SGA) as a nutritional screening tool for cancer patients in an outpatient setting. Nutr Cancer. 2013;65(2):234-239. doi:10.1080/01635581.2013.755554
Cancer Management and Research

\section{Publish your work in this journal}

Cancer Management and Research is an international, peer-reviewed open access journal focusing on cancer research and the optimal use of preventative and integrated treatment interventions to achieve improved outcomes, enhanced survival and quality of life for the cancer patient.
The manuscript management system is completely online and includes a very quick and fair peer-review system, which is all easy to use. Visit http://www.dovepress.com/testimonials.php to read real quotes from published authors. 